\title{
HIGHLIGHTS
}

DIABETES

\section{Novel type 2 diabetes mellitus risk locus near IRS1 is associated with insulin resistance and hyperinsulinemia}

A genome-wide association study has identified a new genetic variant associated with type 2 diabetes mellitus (T2DM) located next to the insulin receptor substrate 1 (IRS1) gene.

The multistage association study consisted of a genome scan of 16,273 single nucleotide polymorphisms (SNPs) in a cohort of 4,977 French individuals and a second study of 28 SNPs-the variants most strongly associated with T2DM-in a Danish cohort of 7,698 individuals.

The investigators found that the $\mathrm{C}$ allele of the SNP rs2943641, located $500 \mathrm{~kb}$ upstream of IRS1, is associated with insulin resistance as well as an increase in pancreatic insulin secretion. "These characteristics distinguish it from the other T2DM risk loci identified to date that have been associated with decreased pancreatic $\beta$-cell function," says Robert Sladek (McGill University, Montreal, Canada).
The effects of the previously unreported risk locus rs2943641 on T2DM-related quantitative traits were further examined in three population-based cohorts of normoglycemic Finnish $(5,824)$, Danish $(5,183)$ and French $(3,351)$ individuals.

\section{These characteristics} distinguish it from the other T2DM risk loci identified to date... 77

The study revealed that the $\mathrm{C}$ allele of rs2943641 was also associated with a reduction in basal levels of IRS1 protein expression in biopsies of human muscle, as well as a decrease in insulininduced IRS1-associated PI3-kinase activity. These findings link rs2943641 to a gene with known key functions in the regulation of glucose metabolism. Sladek and co-workers plan to map the locus and perform cell and molecular biology studies to link the SNP to changes in IRS1 expression and activity. Future studies on whether insulin-treated patients with T2DM who carry the risk variant, especially those with two $\mathrm{C}$ alleles, require more daily insulin than other patients, or whether the SNP can predict the response to specific oral agents, such as metformin or thiazolidinediones, would be of great relevance. "Also, it would be interesting to do long-term outcome studies to investigate if IRS1 variant carriers have a higher risk of cardiovascular events," concludes co-author Oluf Pedersen (Steno Diabetes Center, Gentofte, Denmark)

Linda Koch

Original article Rung, J. et al. Genetic variant near IRS1 is associated with type 2 diabetes, insulin resistance and hyperinsulinemia. Nat. Genet. 41, 1110-1115 (2009) 